\title{
THE INFLUENCE OF FIBRE BENDING ON POLARIZATION-DEPENDENT TWIST SENSOR BASED ON TILTED BRAGG GRATING
}

\author{
Damian Harasim \\ Lublin University of Technology, Faculty of Electrical Engineering and Computer Science, Nadbystrzycka 36D, 20-618 Lublin, Poland \\ $\triangle$ d.harasim@pollub.pl, +48 815384313
}

\begin{abstract}
This paper presents an experimental study on influence of input light polarization on the spectral characteristics of a fibre twist sensor based on Tilted Fiber Bragg Grating (TFBG) with simultaneous application of bending to an optical fibre. The application of proposed measurement stand could provide the ability of transforming the bending to a displacement. The twist measurement was performed by tuning of the sensor illuminating light polarization angle. The spectral parameters of selected cladding mode which are sensitive to the rotation of input light polarization angle have been shown. This paper shows the characteristics of transmittivity and wavelength shift for an incident high-order cladding mode measured with different curvatures of fibre. The dependency of selected cladding mode spectral parameters related with the twist measurement on the influence of temporary bending has been shown. The measurements were performed for two positions of sensing structure refractive index perturbations in relation to the bending direction plane. The experimental results show that the direction of TFBG structure bending has a small influence on the stability of spectral parameters characteristic for twist measurement, assuming that the bending direction is fixed while measurement.
\end{abstract}

Keywords: optoelectronic sensors, tilted bragg grating, sensing technology.

(C) 2017 Polish Academy of Sciences. All rights reserved

\section{Introduction}

Fiber Bragg Grating (FBG) sensors have emerged as a promising and important part of novel measurement systems. The most important advantages of FBG-based sensors are their high sensitivity, good repeatability, small size and immunity to electromagnetic interference [1-4]. Another advantage of measurement systems containing FBG sensing structures is their ability to using several gratings written in a single optical fibre and measuring many parameters at the same time [5]. Because FBGs are inherently sensitive to both strain and temperature, strain sensors based on Bragg grating always require temperature compensation. Some of technological modifications, such as tapering of optical fibre or creating chirp in an FBG structure could be implemented to obtain desirable sensing properties [6-8]. Recently, the Tilted Fiber Bragg Grating (TFBG), which introduces an angle between the grating planes and the fibre cross-section, has been extensively examined due to its distinctive properties in the field of fibre sensing [6]. The most striking effect of TFBG is the strong enhancement of the counter-propagating cladding mode resonances for its tilted grating planes.

The twist sensing is an important requirement in monitoring the health condition in engineering such structures, as buildings and bridges. The most common approach during designing optical fibre twist sensors is to measure the change in circular birefringence caused by twist of fibre, such as circular birefringence is Sagnac loops' twist sensing [9, 10]. The Sagnac-based twist sensors usually suffer from temperature dependence and instability [11]. Recently, the TFBGs which enhance the coupling of light from the core mode with a large number of counter propagating cladding modes, have been used as twist sensors [12]. 
An approach was made of using Long Period Fiber Gratings (LPFGs) to bending measurements by embedding a sensing structure into a composite laminate, but LPFGs present cross-sensitivities between different physical parameters such as pressure, strain and temperature $[13,14]$. The reduction of temperature cross-sensitivity may be obtained by using a hybrid structure consisting of an LPFG and tilted Bragg grating for curvature measurements [15].

Sensing structures based on TFBGs could be applied to obtaining the multi-functionality of a fibre sensor [16]. The bend sensing principles of TFBG spectral response have been demonstrated [17]. Independency from temperature of some TFBG-based sensors could be obtained by the insertion of a short segment of multimode optical fibre between the TFBG structure and a single mode fibre [18].

The spectral response of TFBG influenced by different curvatures and various input light polarization angles has been shown [15], however the dynamics of optical power response to twist could be increased by the application of a stronger tilt angle in the TFBG fabrication process. The enhancement of fibre sensor characteristics is an important prerequisite to manufacture the periodic structures with desirable spectral characteristics. This paper presents a photonic sensing system based on a tilted Bragg grating sensor for measurements of twist/rotation by analysis of optical power and wavelength shift of selected cladding modes with simultaneous influence of fibre curvature changes. The twist measurement was introduced by tuning of the input light polarization angle in relation to planes of periodic variations of refractive index in the fibre core. The spectral response was measured for two different TFBG tilt angles with simultaneous changing of fibre section curvature with written grating. The dependency of twist measurement stability on influence of temporary bending has been examined. Obtaining a double functionality of sensor is an interesting approach to develop.

\section{Sensing principle of TFBG structure}

The transmission spectrum of tilted Bragg grating structure is highly dependent on an introduced tilt angle. The spectral width of cladding modes' dips in the measured spectrum is widening with growth of the angle. The wavelength and transmittivity of some dips and peaks of transmitted TFBG spectrum could be influenced by the input light polarization angle and fibre curvature. The wavelength of a specified cladding mode is determined by the grating tilt angle and effective refractive indexes according to the following expressions [19]:

$$
\begin{gathered}
\lambda^{c o}=\frac{2 n_{\text {eff }}^{c o} \Lambda}{\cos \theta_{\text {TFBG }}}, \\
\lambda_{i}^{c l}=\frac{\left(n_{\text {eff }}^{c o}+n_{\text {eff } i}^{c l}\right) \Lambda}{\cos \theta_{\text {TFBG }}},
\end{gathered}
$$

where: $\Delta \lambda^{c o}$ is a core mode wavelength; $\Delta \lambda_{i}{ }^{c l}$ is a resonance wavelength between the core mode and another one labelled $i$; $n^{c o}$ eff is an effective index of the single mode guided by the core at the wavelength where the resonance is observed; $n^{c l}$ eff $i$ is an effective index of the mode $i$ at the same wavelength; $\Lambda$ is a period of pattern used to create the grating; and $\theta_{\text {TFBG }}$ is a tilt angle of the grating. The reflectivity of individual resonant modes $R_{i}^{c o, c l}$ depends on the modulation of the refractive index according to the following expression [19]:

$$
R_{i}^{c o, c l}=\tanh ^{2}\left\{L C \iint_{-\infty}^{+\infty} \vec{E}^{c o} * \vec{E}^{c l} \Delta n \cos \left(\frac{4 \pi}{\Lambda} z \cos \left(\theta_{T F B G}\right)+y \sin \left(\theta_{T F B G}\right)\right) d x d y\right\},
$$

where: $L$ is a longitude of the grating; $C$ is a proportional constant related to the normalization of the transverse mode fields; $E$ is a transverse component of the electric fields of the modes; 
and $\Delta n$ is a function describing the variation of the refractive index due to the grating crosssection in fibre. The amplitude and wavelength of individual resonant modes could be affected by changes of refractive index values caused by changes of input light polarization angle and TFBG fibre section curvature.

TFBG sensors used in the following experiments were $15 \mathrm{~mm}$ long, written in photosensitive germanium-doped single mode fibres by using the phase mask rotation technique and a BraggStar $\mathrm{KrF}$ excimer pulse laser. The assembled experimental stand provides continuous tuning of the input light polarization angle with simultaneous changing of the bending diameter which affects the fibre section with written TFBG sensing structure. Fig. 1 shows the experimental setup where the twist of input light polarization angle is introduced by an electronically driven rotating table with a half-wave plate. The bending changes were performed by the application of specified radius curvatures to the optical fibre section with written TFBG structure.

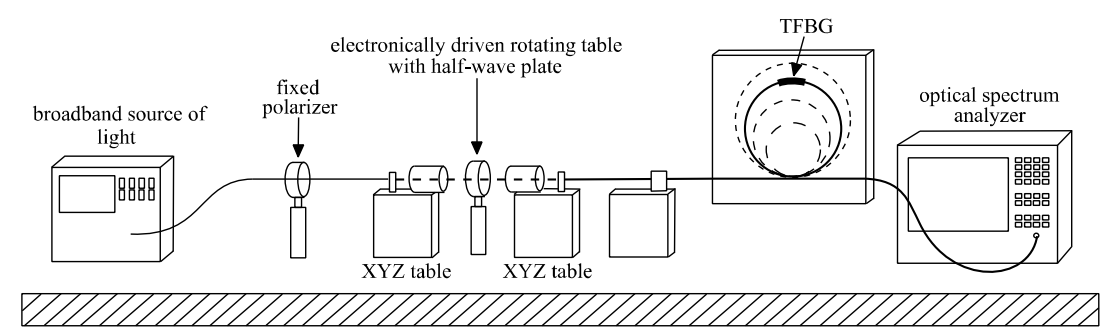

Fig. 1. The experimental stand for measurement of the spectral response of TFBG sensor affected by changes of input light polarization angle and fibre curvature.

\section{Experimental results}

\subsection{Experimental setup and measurement principles}

The experiment was performed with using a tilted Bragg grating with $4^{\circ}$ tilt introduced in the manufacturing process. This tilt angle value provides a good sensor response to changing of the polarization state of input light. Fig. 2 shows a transmission spectrum measured with the introduced input light with P-polarized state. According to Fig. 2b, the P-polarized state means that the polarization plane of illuminated light was parallel to $\mathrm{x}-\mathrm{z}$ plane when the refractive index perturbations in the fibre core are tilted relative to $\mathrm{y}-\mathrm{x}$ plane. In the experimental study the polarization angle was being changed in a $0-90^{\circ}$ range (i.e., from parallel to $\mathrm{x}-\mathrm{z}$ plane to parallel to $\mathrm{y}-\mathrm{z}$ plane).

The changing of input light polarization angle related to planes of tilted periodic refractive index perturbations in fibre core influences on wavelength and transmission coefficient of cladding modes. Actually, the strength of obtained spectral differences depends on the modeorder of considered spectrum dips. Fig. 3. shows the spectral response of chosen cladding mode dips for twisting of input light polarization. Inset (a) concerns the case of high-order cladding mode and inset (b) concerns a case of low-order mode. The wavelengths of depicted spectra are related to characteristic shown in Fig. 2. The relative power difference between 90 and 45 degrees twist for high-order mode is about $40 \%$. The low-order mode is much less sensitive the relative power difference between boundary twist values is about $10 \%$. To designate the influence of curvature applied to TFBG fibre section for twist sensing properties of polarization dependent tilted Bragg grating sensor, the high-order cladding mode with $1535.35 \mathrm{~nm}$ wavelength was chosen. 
a)

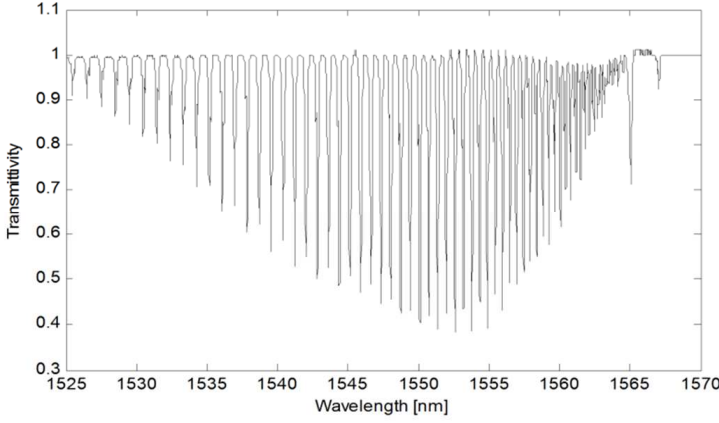

b)

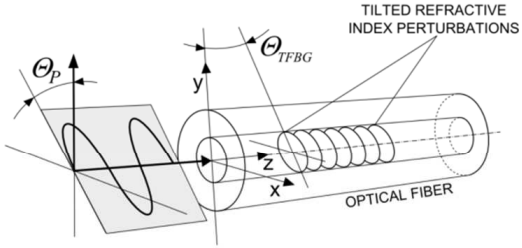

Fig. 2. A transmission spectrum of the tilted Bragg grating twist sensing structure with a $4^{\circ}$ tilt angle in the P-polarized state (a); a schematic diagram of the input light polarization state and bending direction relative to refractive index perturbations $(b)$.

a)

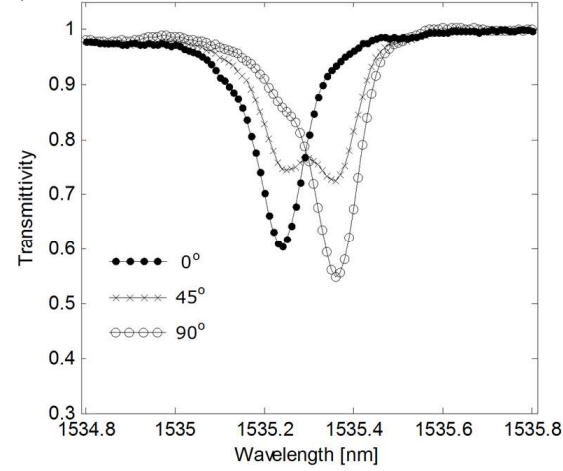

b)

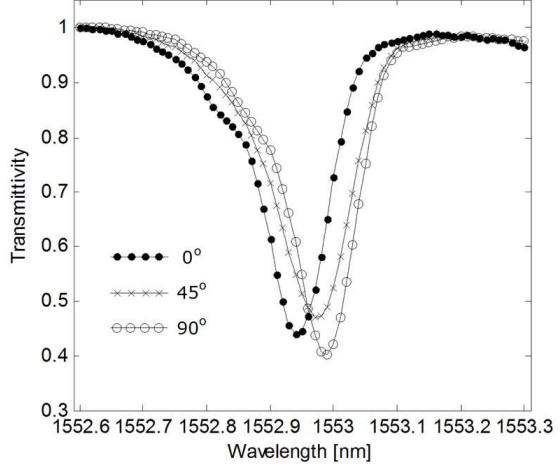

Fig. 3. The spectral response of cladding mode dips in transmission spectrum treated to variable angle of boundary input light polarization: the more sensitive high-order cladding mode (a); low-sensitive low-order cladding mode (b).

The high stability of selected peak spectral properties are desirable in case of obtaining a double functionality of fibre sensor. The optical spectra of selected cladding mode which provides a strong signal response for changes of applied input light polarization twist were measured with using Optical Spectrum Analyzer with $0.02 \mathrm{~nm}$ resolution. The curvatures were performed by strengthening a loop shaped fibre section with written TFBG sensor with using an electronically driven translation stage. Increasing of the distance between stages with fibre clamps causes the decrease of curvature radius.

\subsection{Experimental results for two bending directions}

The influence of bending on the transmission spectrum of TFBG twist sensor was examined for two perpendicular directions of bending in relation to refractive index perturbations in the fibre core. These boundary fibre position rotations have been chosen as representative examples. Fig. 4 shows the boundary cases of bending direction related to TFBG fringes where the curvature plane is parallel to $y-x$ plane. Two created measurement setups are described in the further part of this paper as case I and case II presented in Fig. 4a and Fig. 4b, respectively. 
a)

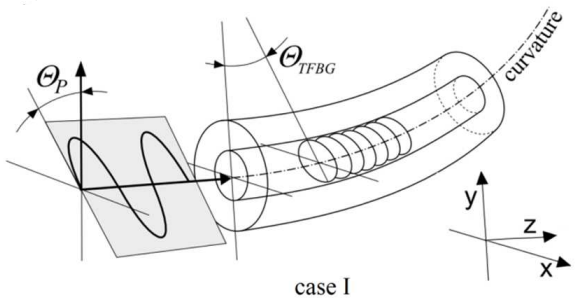

b)

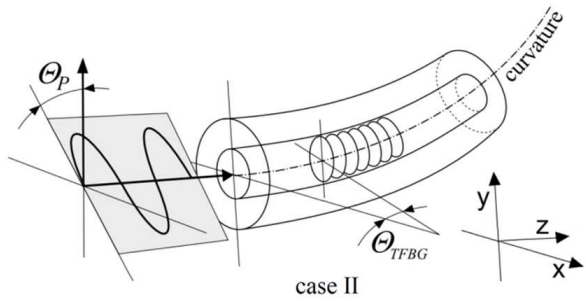

Fig. 4. Views of boundary TBFG sensor angle positions for two cases: TFBG fringes parallel to the bending plane (a); refractive index fringes perpendicular to the bending plane (b).

In both examined cases, the bending direction was specified as parallel to $\mathrm{y}-\mathrm{z}$ plane, i.e. $0^{\circ}$ angle of input light polarization (according to Fig. 4). In case I an optical fibre with a TFBG sensor was positioned as it is shown in Fig. 4a. In case II the fibre was rotated by 90 degrees around its central axis while the bending direction and light polarization planes were the same as previously, which is shown in Fig. 4b. Figs. 5 and 6 show transmission spectra of a highorder cladding mode power dip for three boundary cases: 0,45 and 90 degrees of input light polarization angle chosen as representative examples of twist measurement. The bending of TFBG fibre section was performed in a $28-8 \mathrm{~mm}$ range.

a)

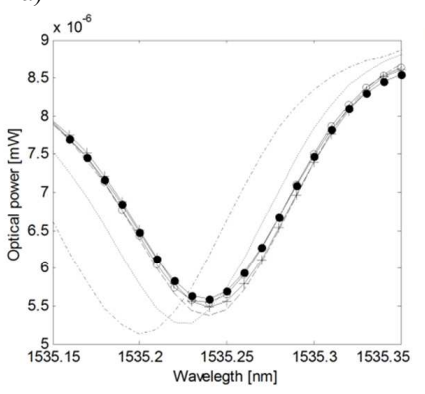

b)

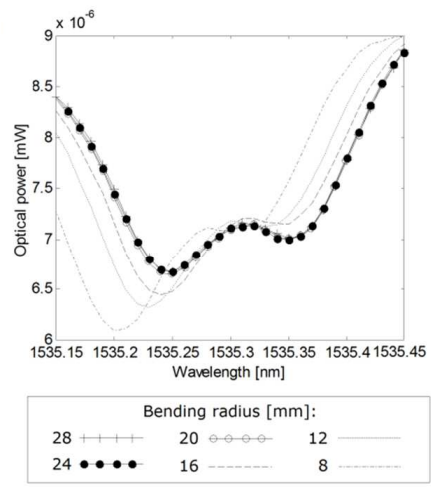

c)

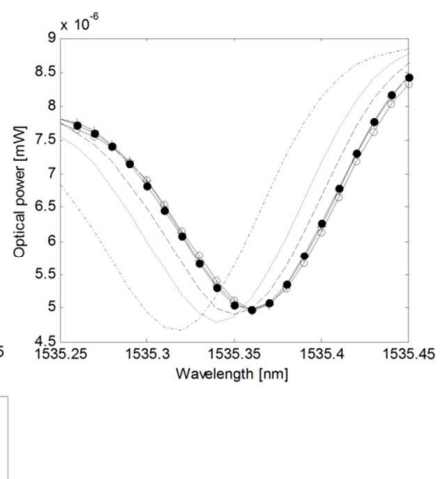

Fig. 5. Spectral responses of a specified cladding mode dip influenced by changing of TFBG fibre bending radius for three boundary cases of input light polarization angle: $0^{\circ}$ (a); $45^{\circ}$ (b); $90^{\circ}$ (c) with the bending direction presented in Fig. $4 \mathrm{a}$.

Figure 5 shows that the spectra of a chosen cladding mode measured for three cases of input light polarization are very stable for curvature diameters decreasing down to $20 \mathrm{~mm}$. The polarization state of illuminating light is not influencing a stability range - in every case the spectrum measured for bending with a $16 \mathrm{~mm}$ radius is shifted to shorter wavelengths. This phenomenon becomes more outstanding when the diameter is shortened down to $8 \mathrm{~mm}$. The high stability of spectral parameters for curvature diameters from 16 to $28 \mathrm{~mm}$ makes reasonable to expect the same behaviour for longer diameters. Fig. 6 shows similar spectra for the same input light polarization angle and bending plane dependencies, measured for the TFBG sensor rotated by 90 degrees around the central axis, which is shown in Fig. $4 \mathrm{~b}$. The line styles represent spectra measured for the TFBG structure curved with the same radiuses as shown in Fig. 5, respectively. 
a)

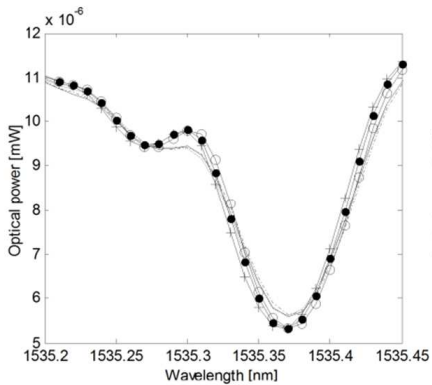

b)

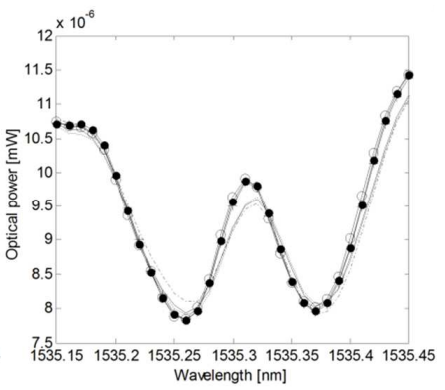

c)

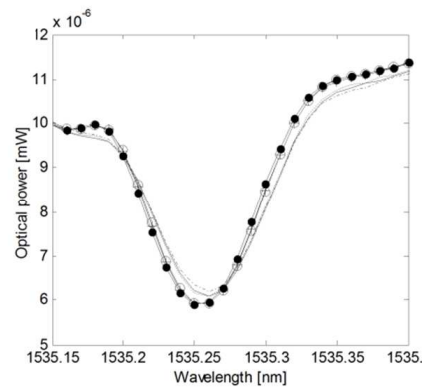

Fig. 6. Spectral responses of the same high-order cladding mode influenced by changing of TFBG fibre bending radius for three boundary cases of input light polarization angle: $0^{\circ}$ (a); $45^{\circ}$ (b); $90^{\circ}$ (c) with the bending direction presented in Fig. $4 \mathrm{~b}$.

The spectral characteristics showed in Fig. 6 prove that the strengthening of TFBG sensor curvature has a very weak influence on the cladding mode transmission characteristics. It should be noticed that the spectra measured for two fibre position cases and for the same input light polarization state, have different shapes. Therefore, the fibre sensing section has to be fixed in one rotation state. Fig. 7 and Fig. 8 show characteristics of transmission and wavelength shift of a specified cladding mode power dip in a function of strengthened bending radius. The plots marked as case I and case II refer to the spectral characteristics depicted in Fig. 5 and Fig. 6, respectively.

case I

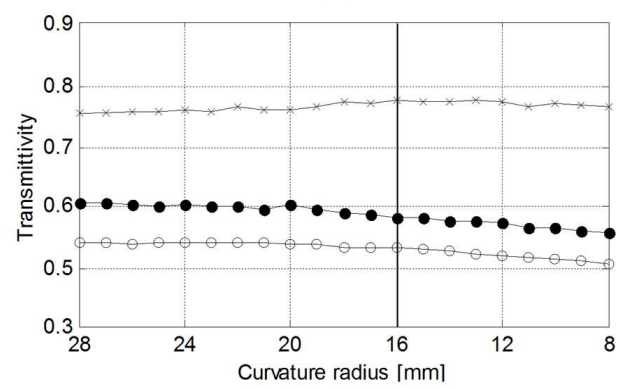

case II

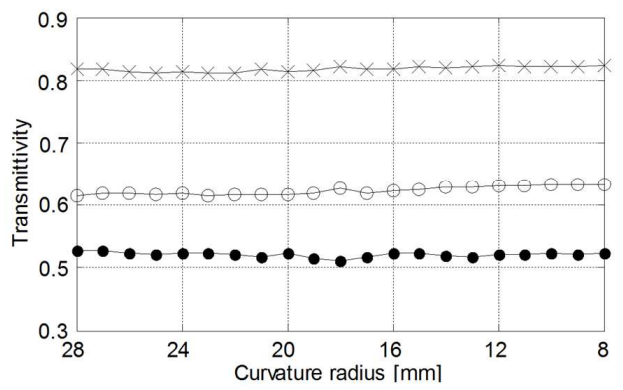

Fig. 7. Stability plots of a chosen high-order cladding mode transmission coefficient in a function of a curvature radius decrease.
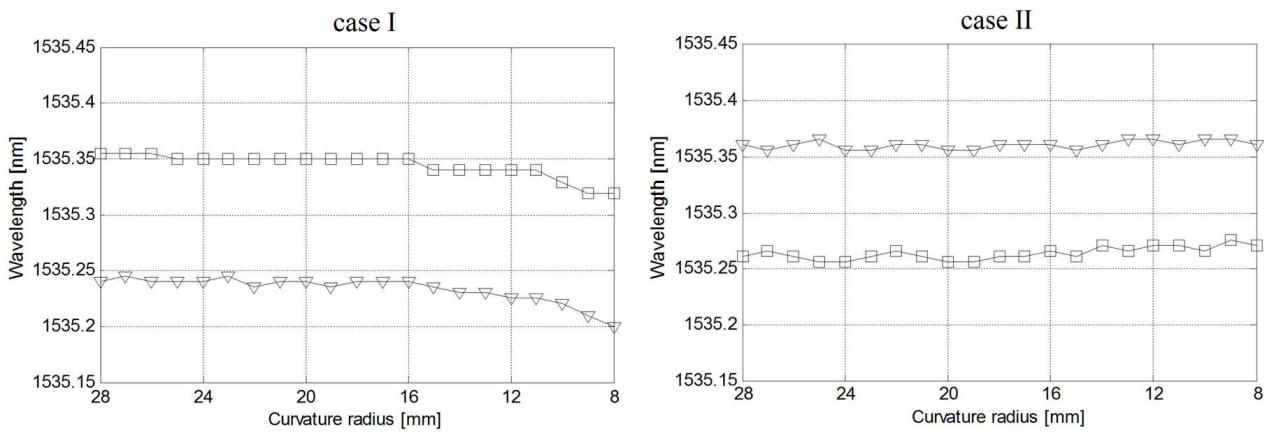

Fig. 8. Characteristics of a specified high-order cladding mode wavelength stability in a function of a decrease of TFBG fibre section curvature radius. 
The above graphs prove that a decrease of curvature diameter of fibre section with TBFG has a weak influence on transmissivity and wavelength of a selected high-order cladding mode spectrum. The spectral parameters of selected cladding mode of sensing structure positioned in the experimental stand defined as case I show the greater sensitivity for small curvature radiuses. The mode dip transmittivity and wavelength begin to shift from $16 \mathrm{~mm}$ radius to a strengthened bend. In case II of refractive index perturbation position, the transmission spectrum of a selected high-order cladding mode is more stable for small bending radiuses. Due to this conditions, Table 1 contains coefficient values calculated for transmittivity and wavelength in a $28-16 \mathrm{~mm}$ range of curvature radiuses. Table 1 shows the maximum differences of transmission and wavelength of a selected cladding mode spectrum measured for two cases of sensor position and three different input light polarization angles - for bending radiuses in a $28-16 \mathrm{~mm}$ range.

Table 1. The maximum values of differences of transmission and wavelength of a selected cladding mode spectrum for two cases of sensor position and three different input light polarization angles

(the bending radius in a $28-16 \mathrm{~mm}$ range).

\begin{tabular}{|c|c|c|c|c|c|c|}
\hline & \multicolumn{3}{|c|}{ case I } & \multicolumn{3}{c|}{ case II } \\
\hline Input light polarization & 0 & 45 & 90 & 0 & 45 & 90 \\
\hline Transmission & 0,0136 & 0,0373 & 0,0289 & 0,0164 & 0,0102 & 0,0132 \\
\hline Wavelength shift [nm] & 0,01 & 0,014 & 0,016 & 0,012 & 0,08 & 0,016 \\
\hline
\end{tabular}

\section{Conclusions}

In this paper the stability characteristics of selected spectral parameters of TFBG-based sensor for simultaneous twist and bending are shown. The twist sensing is based on the sensor illuminating light polarization dependencies of tilted Bragg gratings. Two different positions of fibre in relation to the bending direction plane - denoted as case I and case II - were examined. Increasing a grating tilt angle could significantly improve the dynamics of highorder mode spectral response to a change of input light polarization plane angle. In addition the strongest tilt angle provides different changes of spectral characteristics when the sensing structure is influenced by bending with evolving curvatures.

The measurement results show that the selected high-order cladding mode of a 4 degree tilted TFBG sensing structure has a high transmission coefficient and dip wavelength stability while being curved with strengthened radiuses. The characteristics shown in Fig. 5 and Fig. 6 show the spectra of the same cladding mode measured for the same illuminating light polarization angle but for different fibre positions, which is presented in Fig. 4. The fibre was rotated by 90 degrees around its central axis to obtain two boundary positions of planes of TFBG fringes related to the bending direction. Based on the shown spectra, it is clear that for both fibre positions in specified ranges of curvature radiuses the spectra show a high stability. Comparison of spectral characteristics obtained for the same curvature diameters but with different fibre positions shows that in a real measurement system of two quantities, the fibre sensing structure has to be kept in a position which guarantees no rotation around its central axis. Uncontrolled rotating of fibre could also destructively influence the twist sensing abilities. However, the starting fibre angle arrangement is straightforward when we analyse the stability of measured cladding mode parameters - fibre just has to be prevented from uncontrolled rotating. The measurement results for both boundary cases of sensing structure position in relation to the bending direction plane show that in both cases the measured spectra were very stable when the loop with a sensing TFBG was strengthened, decreasing its curvature. The high stability of spectral parameters for curvature diameters from 16 to $28 \mathrm{~mm}$ makes 
reasonable to expect the same behaviour for longer diameters. The improvement of TFBG sensor twist sensing dynamics by the application of a greater internal tilt could be employed, with no destructive influence on the bending-dependent stability of high-order twist-sensitive cladding modes' spectral characteristics.

\section{References}

[1] Kisała, P., Cieszczyk, S. (2015). Method of simultaneous measurement of two direction force and temperature using FBG sensor head. Applied Optics, 54(10), 2677-2687.

[2] Harasim, D., Gulbahar, Y. (2015). Improvement of FBG peak wavelength demodulation using digital signal processing algorithms. Proc. SPIE, 9662-966212.

[3] Kisała, P. (2012). Metrological conditions of strain measurement optoelectronic method by the use of fibre Bragg gratings. Metrol. Meas. Syst., 19(3), 471-480.

[4] Schroeder, K., Ecke, W., Willsch, R. (2009). Optical fiber Bragg grating hydrogen sensor based on evanescent-field interaction with palladium thin-fim transducer. Optics and Lasers in Engineering, 47, $1018-1022$

[5] Kisała, P. (2012). Generation of a zone chirp in uniform Bragg grating as a way of obtaining double functionality of a sensor. Metrol. Meas. Syst., 19(4), 727-738.

[6] Osuch, T., Markowski, K., Manujło, A., et. al. (2016). Coupling independent fiber optic tilt and temperature sensor based on chirped tapered fiber Bragg grating in double-pass configuration. Sensors And Actuators A Physical, 252, 76-81.

[7] Markowski, K., Jędrzejewski, K., Marzęcki, M., Osuch, T. (2017). Linearly chirped tapered fiber-Bragggrating-based Fabry-Perot cavity and its application in simultaneous strain and temperature measurement. Optics Letters, 42(7), 1464-1467.

[8] Osuch, T., Markowski, K., Jędrzejewski, K. (2016). Fiber-Optic Strain Sensors Based on Lineaely Chirped Tapered Fiber Bragg Gratings With Tailored Intrinsic Chirp. IEEE Sensors J., 16(20), 7508-7514.

[9] Silva., R.M., Ferreira, M.S., Frazao, O. (2012). Temperature independent torsion sensor using a highbirefringent Sagnac loop interferometer. Optics Communications, 285, 1167-1170.

[10] Zu, P., Chan, C.C., Jin, Y.X., Gong, T.X., Zhang, Y.F., Chen, L.H., Dong, X.Y. (2011). A temperature insensitive twist sensor by using low-birefringence photonic-crystal-fiber-based Sagnac interferometer. IEEE Photon. Technol. Lett., 23, 920-922.

[11] Lu, Y., Shen, C., Chen, D., Chu, J., Wang, Q., Dong, X. (2014). Highly sensitive twist sensor based on tilted fiber Bragg grating of polarization-dependent properties. Optical Fiber Technology, 20(5), 491-494.

[12] Chen, X., Zhou, K.M., Zhang, L., Bennion, I. (2006). In-fiber twist sensor based on a fiber Bragg grating with 81 tilted structure. IEEE Photon. Lett., 18, 2596-2598.

[13] Tan, K.M., Chan, C.C., Tjin, S.C., Dong, X.Y. (2006). Embedded long-period fiber grating bending sensor. Sensors and Actuat. A-Phys., 125, 267-272.

[14] Bhatia, V., Vensegsarkar, A.M. (1996). Optical fiber long-period grating sensors. Opt. Lett., 21, 692-694.

[15] Shao, L.Y., Laronche, A., Smietana, M., Mikulic, P., Bock, W.J., Albert, J. (2010). Highly sensitive bend sensor with hybrid long-period and tilted fiber Bragg grating. Opt. Commun., 283, 2690-2694.

[16] Osuch, T., Jurek, T., Markowski, K. (2016). Simultaneous measurement of liquid level and temperature using tilted fiber Bragg greting. IEEE Sensors Journal, 16(5), 1205-1209.

[17] Kisała, P., Harasim, D., Mroczka, J. (2016). Temperature-insensitive simultaneous rotation and displacement (bending) sensor based on tilted fiber Bragg grating. Optics Express, 24(26), 29922-29929.

[18] Jin, Y.X., Chan, C.C., Dong, X.Y., Zhang, Y.F. (2009).Temperature-independent bending sensor with tilted fiber Bragg grating interacting with multimode fiber. Opt. Commun., 282, 3905-3907.

[19] Albert, J., Shao, L.Y., Caucheteur, C. (2013). Tilted fiber Bragg grating sensors. Laser \& Photonics Reviews, $7(1), 83-108$ 